\title{
THE PROBLEM OF DATA ASSIMILATION FOR SOIL WATER MOVEMENT
}

\author{
François-Xavier Le Dimet ${ }^{1}$, Victor Petrovich Shutyaev ${ }^{2}$, \\ JIAFENG WANG ${ }^{3}$ AND MU MU ${ }^{3}$
}

\begin{abstract}
The soil water movement model governed by the initial-boundary value problem for a quasilinear 1-D parabolic equation with nonlinear coefficients is considered. The generalized statement of the problem is formulated. The solvability of the problem is proved in a certain class of functional spaces. The data assimilation problem for this model is analysed. The numerical results are presented.
\end{abstract}

Mathematics Subject Classification. 65K10.

Received October 2, 2002. Revised November 4, 2003.

\section{INTRODUCTION}

Today, the concept of "climate system" has been accepted by more and more people. Change of climate is the synthetic results of the individual change of atmosphere, ocean, land surface and sea-ice and their interactions. Many climate models are being used for research. Compared with the atmosphere and ocean, the research on the land surface process needs development urgently so as to improve the whole quality of the climate model. With the implementation of the outside observation experiments such as HAPEX, etc., the land surface data are given, which is of great help to the land surface process research on two aspects: one is the recognition of detail physical process of changes on the land surface, the other is the model construction.

The land surface physical process is the interaction between the land surface and atmosphere through the material and energy's input and output, which can take effect on the climate change. It mainly refers to the exchange of water and the heat flux. These processes are divided into two types according to the land surface cover: one is the interaction of bare soil and the atmosphere, the other is of the plant and atmosphere. In this paper, we focus on the water movement in the even, isothermal and unsaturated soil, which takes evaporation as the driven force.

In the land surface process, the exchanges of water and the heat flux are connected together. The solar radiation heats the soil surface, which improve the evaporation and the soil water moving. The evaporated vapor enters air and also heats air when it condenses. So, in the water-cycle on the land surface (evaporation, precipitation, run-off and infiltration), the soil water movement with evaporation's effect plays the important role.

\footnotetext{
Keywords and phrases. Variational data assimilation, soil water movement, quasilinear parabolic problem, solvability, numerical analysis.

${ }^{1}$ LMC-IMAG, 38041 Grenoble Cedex 9, France.

2 Institute of Numerical Mathematics, Russian Academy of Sciences, Russia.

${ }^{3}$ LASG, Institute of Atmospheric Physics, Chinese Academy of Sciences, Beijing, 100029, China.
} 
As a lower boundary to the atmosphere, when this process is coupled into a climate model, the prediction of the soil wetness should be done. This is an initial-value problem. The quality of the initial field is crucial to the forecast result. The soil water movement is a strongly nonlinear process, and the data on soil wetness is scarce, so to take the most use of this limited data and provide a good initial field to modelling is a very prominent problem. Fortunately, variational assimilation is a promising way to realize this.

Presently, the problems of data assimilation are being studied on the basis of adjoint equations by many researchers $[2,4,15,18,25,29]$. In this paper, we consider the data assimilation problem for the soil water movement model, and using the technique developed in $[2,15,29]$, give the numerical analysis of the problem.

In the paper, the statement of the problem is given in Section 2. In Section 3, the solvability is proved. Data assimilation for this model is discussed in Section 4. Some numerical results are presented in Section 5.

\section{Statement of the Problem}

In this model, the soil water movement in the horizontal direction is not considered. So, the model is a $z-t$ model, and it is governed by the following quasilinear parabolic equation [16]:

$$
\frac{\partial \theta}{\partial t}=\frac{\partial}{\partial z}\left(D(\theta) \frac{\partial \theta}{\partial z}\right)-\frac{\partial K(\theta)}{\partial z}
$$

with the nonlinear coefficients

$$
\begin{gathered}
D(\theta)=\frac{-b \Phi_{s} K_{s}}{\theta_{s}}\left(\frac{\theta}{\theta_{s}}\right)^{b+2}, \\
K(\theta)=K_{s}\left(\frac{\theta}{\theta_{s}}\right)^{2 b+3},
\end{gathered}
$$

where $\theta=\theta(t, z)$ is the soil wetness, $z$ is the vertical direction. In this model, downward is positive and the depth of soil is $L, t$ is the time coordinate. The functions $D(\theta)$ and $K(\theta)$ are the diffusion coefficient and conductivity, respectively. The subscript " $s$ " means "saturation", the constants $\Phi_{s}, K_{s}, \theta_{s}$ are corresponding soil potential, conductivity and wetness when soil is saturated, $K_{s}, \theta_{s}>0, \Phi_{s}<0, b$ is a positive soil parameter. The coefficients $D(\theta)$ and $K(\theta)$ may be defined also by other formulas, for example, [16]:

$$
D(\theta)=D_{0} \exp \left(-\beta\left(\theta_{0}-\theta\right)\right), K(\theta)=K_{s} \exp \left(-\beta\left(\theta_{s}-\theta\right)\right), \beta>0 .
$$

The initial condition is:

and the boundary conditions are:

$$
t=0, \quad 0 \leq z \leq L, \quad \theta(0, z)=\theta_{0}(z)
$$

$$
\begin{gathered}
t>0, \quad z=0, \quad D(\theta) \frac{\partial \theta}{\partial z}-K(\theta)=E_{p}, \quad \text { if } \quad \theta \geq \theta_{k} \\
t>0, \quad z=0, \quad D(\theta) \frac{\partial \theta}{\partial z}-K(\theta)=\frac{\theta}{\theta_{k}} E_{p}, \quad \text { if } \quad 0<\theta<\theta_{k} \\
t>0, \quad z=L, \quad \theta(t, L)=\theta_{1}(t),
\end{gathered}
$$

where $E_{p}$ is the potential evaporation controlled by the air condition, $\theta_{k}$ is a parameter which gives the soil's capability of holding water. It is smaller than the saturated wetness. In physics, when $\theta \geq \theta_{k}$, it means the soil is wet enough, so evaporation is wholly controlled by air. While $\theta<\theta_{k}$, the soil wetness also becomes a factor which decides the evaporation. In this phase, evaporation should be related to wetness. Here, for simplicity, linear relation is taken. According to this fact, the upper boundary condition in the model is divided into two parts. The functions $\theta_{0}(z), \theta_{1}(t)$ are assumed to be prescribed.

Assume the wetness observation in a time interval be available. To assimilate its initial state, the cost function is formulated:

$$
J\left(\theta_{0}\right)=\int_{0}^{T}\left\langle\left(\theta\left(\theta_{0}\right)-\theta^{o}\right), w(t)\left(\theta\left(\theta_{0}\right)-\theta^{o}\right)\right\rangle \mathrm{d} t,
$$


where $(0, T)$ is the assimilating interval, $\theta^{\circ}$ is the observational data, and $w(t)$ is the weight factor. The goal is to find $\theta_{0}^{*}$ that makes $J\left(\theta_{0}\right)$ get the minimum. This is an optimal control problem which may be solved by the gradient method. The gradient of $J$ with respect to $\theta_{0}$ may be calculated by the adjoint method, following $[2,4,15,18,25,29]$.

\section{Solvability of The INITIAL-BOUNDARY VALUE PROBLEM}

Consider the initial-boundary value problem for quasilinear 1-D parabolic equation of the form:

$$
\begin{gathered}
\frac{\partial \theta}{\partial t}-\frac{\partial}{\partial z} D \frac{\partial \theta}{\partial z}+\frac{\partial K}{\partial z}=f(t, z), \quad t \in(0, T), z \in(0, L) \\
\left.\theta\right|_{t=0}=\theta_{0}(z),\left.\theta\right|_{z=L}=\theta_{1}(t),\left.\left(D \frac{\partial \theta}{\partial z}-K\right)\right|_{z=0}=\phi(t),
\end{gathered}
$$

where $\theta=\theta(t, z)$ is the unknown function, the coeffients $D=D(\theta), K=K(\theta)$, and the functions $f(t, z), \theta_{0}(z)$, $\theta_{1}(t), \phi(t)$ are assumed to be prescribed, $t \in(0, T), z \in(0, L), L, T<\infty$.

The issues related to the statement, solvability, and regularity properties of the quasilinear parabolic problems have been reported by many authors, the well-known monographs and surveys $[3,9-12,19,20,24,27,28]$ among them. The results on existence and uniqueness of weak solutions of the initial-boundary value problems for quasilinear parabolic equations in the general form are given in the monograph [11].

In this paper, we consider the initial-boundary value problem for the quasilinear parabolic equation of the form (7) with the coefficients $D(\theta), K(\theta)$ depending on the solution, and with nonlinear boundary condition at $z=0$. The generalized statement of the problem is formulated. The existence and uniqueness of the weak solution is proved in a specific class of functional spaces.

\subsection{Transformation of the problem. Functional spaces. Generalized formulation}

By the Kirchoff transformation

$$
u=\int_{0}^{\theta} D(s) \mathrm{d} s
$$

the problem (7) is reduced to the form:

$$
\begin{aligned}
& C(u) \frac{\partial u}{\partial t}-\frac{\partial^{2} u}{\partial z^{2}}+\frac{\partial \tilde{K}(u)}{\partial z}=f(t, z), \quad t \in(0, T), z \in(0, L) \\
& \left.u\right|_{t=0}=u_{0}(z),\left.u\right|_{z=L}=u_{1}(t),\left.\left(\frac{\partial u}{\partial z}-\tilde{K}(u)\right)\right|_{z=0}=\phi(t),
\end{aligned}
$$

where $C(u)=1 / D(\theta), \tilde{K}(u)=K(\theta), u_{i}=\int_{0}^{\theta_{i}} D(s) \mathrm{d} s, i=0,1$. Below, we consider the problem (8), and the functions $C(u), \tilde{K}(u)$ are assumed to be measurable, and bounded almost everywhere, and

$$
0<C_{0} \leq C(u) \leq C_{1}<\infty,|\tilde{K}(u)| \leq k_{1}, k_{1}=\text { const }>0 .
$$

We assume also that $\tilde{K}$ is differentiable almost everywhere and

$$
\left|\frac{\partial \tilde{K}}{\partial u}\right| \leq k_{2}, k_{2}=\text { const }>0 .
$$


We rewrite the problem (8) in the form:

$$
\begin{gathered}
\frac{\partial u}{\partial t}-\alpha \frac{\partial^{2} u}{\partial z^{2}}+(\alpha C(u)-1) \frac{\partial u}{\partial t}+\alpha \frac{\partial \tilde{K}(u)}{\partial z}=\alpha f(t, z), \quad t \in(0, T), z \in(0, L) \\
\left.u\right|_{t=0}=u_{0},\left.u\right|_{z=L}=u_{1},\left.\left(\frac{\partial u}{\partial z}-\tilde{K}(u)\right)\right|_{z=0}=\phi
\end{gathered}
$$

where $\alpha=2 /\left(C_{0}+C_{1}\right)$.

Let $\bar{u}$ be the solution of the linear problem:

$$
\begin{aligned}
& \frac{\partial \bar{u}}{\partial t}-\alpha \frac{\partial^{2} \bar{u}}{\partial z^{2}}=\alpha f, \quad t \in(0, T), z \in(0, L) \\
& \left.\bar{u}\right|_{t=0}=u_{0},\left.\quad \bar{u}\right|_{z=L}=u_{1},\left.\left(\frac{\partial \bar{u}}{\partial z}\right)\right|_{z=0}=0
\end{aligned}
$$

It is well-known that for sufficiently regular functions $u_{0}, u_{1}, f$, there exits a unique solution to the problem (12). Subtracting (12) from (11) we obtain the problem for the remainder $\tilde{u}=u-\bar{u}$ :

$$
\begin{gathered}
\frac{\partial \tilde{u}}{\partial t}-\alpha \frac{\partial^{2} \tilde{u}}{\partial z^{2}}+(\alpha C(\bar{u}+\tilde{u})-1) \frac{\partial(\bar{u}+\tilde{u})}{\partial t}+\alpha \frac{\partial \tilde{K}(\bar{u}+\tilde{u})}{\partial z}=0, \quad t \in(0, T), z \in(0, L) \\
\left.\tilde{u}\right|_{t=0}=0,\left.\tilde{u}\right|_{z=L}=0,\left.\left(\frac{\partial \tilde{u}}{\partial z}-\tilde{K}(\bar{u}+\tilde{u})\right)\right|_{z=0}=\phi
\end{gathered}
$$

Below, assuming that $\bar{u}$ is known, we will investigate the problem (13). To introduce the generalized statement of the problem and its operator formulation, let us consider the space $X=L_{2}(0, L)$ of real-valued functions that are Lebesgue square integrable on $(0, L)$, and the space $X_{1}=\left\{u(z) \in W_{2}^{2}(0, L):\left.u\right|_{z=L}=0,\left.\left(\frac{\partial u}{\partial z}\right)\right|_{z=0}=0\right\}$, where $W_{2}^{2}(0, L)$ is the Sobolev space of functions of $L_{2}(0, L)$ that have square-integrable first and second derivatives with respect to $z$. Let us introduce also the spaces $Y=L_{2}(0, T ; X), Y_{1}=L_{2}\left(0, T ; X_{1}\right)$ of abstract functions $v(t)$ with values in $X, X_{1}$, respectively, and the spaces

$$
W=\left\{v \in Y_{1}: \frac{\mathrm{d} v}{\mathrm{~d} t} \in Y\right\}, W_{T}=\left\{v \in W:\left.v\right|_{t=T}=0\right\} .
$$

For simplicity, we omit the sub-index in the scalar product and assume that $(\cdot, \cdot)_{L_{2}(0, T ; X)}=(\cdot, \cdot)$. Let us introduce the following generalized statement of the problem (13).

Definition 3.1. The function $\tilde{u} \in Y$ is said to be the weak solution of the problem (13) if the relation holds:

$$
\begin{aligned}
-\left(\tilde{u}, \frac{\partial w}{\partial t}\right)-\alpha\left(\tilde{u}, \frac{\partial^{2} w}{\partial z^{2}}\right)+\left(\tilde{C}(\tilde{u}+\bar{u}), \frac{\partial w}{\partial t}\right) & -\alpha\left(\tilde{K}(\tilde{u}+\bar{u}), \frac{\partial w}{\partial z}\right) \\
& =-\left.\int_{0}^{L} \tilde{C}\left(u_{0}\right) w\right|_{t=0} \mathrm{~d} z-\left.\alpha \int_{0}^{T} \phi(t) w\right|_{z=0} \mathrm{~d} t, \quad \forall w \in W_{T}
\end{aligned}
$$

where $\tilde{C}(u)=u-\alpha \tilde{C}_{1}(u), \tilde{C}_{1}(u)=\int_{0}^{u} C(s) \mathrm{d} s$.

Remark 3.1. The equality (14) is obtained by multiplying the equation (13) scalarly by $w$, integrating by parts and taking into account the boundary conditions. 


\subsection{Operator formulation of the problem. Properties of nonlinear operator}

We denote by $A: Y \rightarrow Y$ the linear operator with the domain of definition $D(A)=Y_{1}$, defined by the formula:

$$
A \varphi=-\alpha \frac{\partial^{2} \varphi}{\partial z^{2}}, \quad \varphi \in Y_{1}
$$

The norm in $W_{T}$ may be taken as follows [20]:

$$
\|w\|_{W_{T}}=\left\|-\frac{\mathrm{d} w}{\mathrm{~d} t}+A^{*} w\right\|_{Y},
$$

where the operator $A^{*}: Y \rightarrow Y$ is adjoint to $A$; in the case under consideration, $A^{*}$ is identical to $A$. (Note that the space $X^{*}$ is identified with $X$, and $\left(L_{2}(0, T ; X)\right)^{*} \equiv L_{2}(0, T ; X)$.)

The problem (14) may be written in the operator form: find $\tilde{u} \in Y$ such that

$$
-\left(\tilde{u}, \frac{\partial w}{\partial t}\right)+(\tilde{u}, A w)+(F(\tilde{u}+\bar{u}), w)=q(w), \forall w \in W_{T},
$$

where $q(w)=-\left.\int_{0}^{L} \tilde{C}\left(u_{0}\right) w\right|_{t=0} \mathrm{~d} z-\left.\alpha \int_{0}^{T} \phi(t) w\right|_{z=0} \mathrm{~d} t$, and $F(\tilde{u})$ is the nonlinear operator defined by the formula:

$$
(F(u), w)=\left(\tilde{C}(u), \frac{\partial w}{\partial t}\right)-\alpha\left(\tilde{K}(u), \frac{\partial w}{\partial z}\right), w \in W_{T} .
$$

Remark 3.2. If $u_{0} \in L_{2}(0, L), \phi \in L_{2}(0, T)$, then

$$
|q(w)| \leq \sqrt{T}\left\|\tilde{C}\left(u_{0}\right)\right\|_{L_{2}(0, L)}\left\|\frac{\partial w}{\partial t}\right\|_{Y}+\alpha \sqrt{L}\|\phi\|_{L_{2}(0, T)}\left\|\frac{\partial w}{\partial z}\right\|_{Y},
$$

and the integrals in the right-hand side of (14), (15) have sense for $w \in W_{T}$.

Let us analyze the properties of the operator $F$.

Lemma 3.1. The operator $F$ is bounded from $Y$ into $W_{T}^{*}$.

Proof. By virtue of (9), we get $|\tilde{C}(u)| \leq\left(1+\alpha C_{1}\right)|u|$, then

$$
\left|\left(\tilde{C}(\tilde{u}), \frac{\partial w}{\partial t}\right)\right| \leq\left(1+\alpha C_{1}\right)\|u\|_{Y}\left\|\frac{\partial w}{\partial t}\right\|_{Y} .
$$

Following [26], it is readily seen that

$$
\left\|\frac{\mathrm{d} w}{\mathrm{~d} t}\right\|_{Y} \leq\|w\|_{W_{T}}, \quad\|A w\|_{Y} \leq\|w\|_{W_{T}}
$$

Since

$$
\frac{\partial \psi}{\partial z}=-\int_{z}^{L} \frac{\partial^{2} \psi}{\partial z^{2}} \mathrm{~d} z, \psi \in W_{T}
$$

then, from (9), we get

$$
\left|\left(\tilde{K}(u), \frac{\partial w}{\partial z}\right)\right| \leq k_{1} \sqrt{T L} \frac{L}{2}\left\|\frac{\partial^{2} w}{\partial z^{2}}\right\|_{Y}=k_{1} \sqrt{T L} \frac{L}{2 \alpha}\|A w\|_{Y} .
$$


From (17)-(19), we obtain the inequality

$$
|(F(u), w)| \leq\left[\left(1+\alpha C_{1}\right)\|u\|_{Y}+k_{1} \sqrt{T L} \frac{L}{2}\right]\|w\|_{W_{T}}, u \in Y, w \in W_{T},
$$

which implies that $F: Y \rightarrow W_{T}^{*}$ is bounded.

Lemma 3.2. At any point $u \in Y$, the operator $F$ has the Gateaux derivative $F^{\prime}(u)$ defined by the formula

$$
\left(F^{\prime}(u) v, w\right)=\left(v, \frac{\partial w}{\partial t}\right)-\alpha\left(C(u) v, \frac{\partial w}{\partial t}\right)-\alpha\left(\frac{\partial \tilde{K}}{\partial u} v, \frac{\partial w}{\partial z}\right), v \in Y, \forall w \in W_{T}
$$

The operator $F^{\prime}(u)$ is bounded from $Y$ into $W_{T}^{*}$, and

$$
\left\|F^{\prime}(u) v\right\|_{W_{T}^{*}} \leq k\|v\|_{Y}
$$

where $k=\sup _{t, z}|1-\alpha C(u)|+k_{2} L \leq \frac{C_{1}-C_{0}}{C_{1}+C_{0}}+k_{2} L$, and the constants $C_{0}, C_{1}, k_{2}$ are defined in $(8,9)$.

Proof. The existence of $F^{\prime}(u)$ is proved by using the definition of the Gateaux derivative, following [21]. From (20), we get

$$
\begin{gathered}
\left|\left(v, \frac{\partial w}{\partial t}\right)-\alpha\left(C(u) v, \frac{\partial w}{\partial t}\right)\right| \leq \sup _{t, z}|1-\alpha C(u)| \cdot\|v\|_{Y}\left\|\frac{\partial w}{\partial t}\right\|_{Y}, \\
\left|\left(\frac{\partial \tilde{K}}{\partial u} v, \frac{\partial w}{\partial z}\right)\right| \leq k_{2}\|v\|_{Y}\left\|\frac{\partial w}{\partial z}\right\|_{Y} \leq k_{2} L\|v\|_{Y}\left\|\frac{\partial^{2} w}{\partial z^{2}}\right\|_{Y}=\frac{k_{2} L}{\alpha}\|v\|_{Y}\|w\|_{W_{T}},
\end{gathered}
$$

which, in view of (8), implies the estimate (21).

Remark 3.3. Note that the right-hand side $q(w)$ of equation (15) is a linear bounded functional on $W_{T}$, that is, $q \in W_{T}^{*}$. In view of this fact, the equation (15) may be treated as an operator equation in $W_{T}^{*}$.

\subsection{The linear problem}

Consider the linear problem, obtained from (15) for $F \equiv 0$, with some right-hand side $g \in W_{T}^{*}$ : find $R \in Y$ such that

$$
-\left(R, \frac{\mathrm{d} w}{\mathrm{~d} t}\right)+(R, A w)=(g, w) \quad \forall w \in W_{T} .
$$

Lemma 3.3. For any $g \in W_{T}^{*}$, there exists a unique solution $R \in Y$ to the problem (22) such that

$$
\|R\|_{Y} \leq\|g\|_{W_{T}^{*}}
$$

Proof. The existence is proved by following the arguments of [21] with use of the Lax-Milgram lemma. To prove the estimate (23), note that (22) entails

$$
\left(R,-\frac{\mathrm{d} w}{\mathrm{~d} t}+A w\right)=(g, w) \quad \forall w \in W_{T}
$$

It is known [20] that the space $W_{T}$ is isomorphic to $Y$ by virtue of the equality

$$
v=-\frac{\mathrm{d} w}{\mathrm{~d} t}+A w, w \in W_{T}, v \in Y .
$$


Then,

$$
\|R\|_{Y}=\sup _{v \in Y} \frac{(R, v)}{\|v\|_{Y}}=\sup _{w \in W_{T}} \frac{\left(R,-\frac{\mathrm{d} w}{\mathrm{~d} t}+A w\right)}{\left\|-\frac{\mathrm{d} w}{\mathrm{~d} t}+A w\right\|_{Y}}=\sup _{w \in W_{T}} \frac{(g, w)}{\|w\|_{W_{T}}}=\|g\|_{W_{T}^{*}} .
$$

The lemma is proved.

\subsection{Solvability of nonlinear problem}

In this subsection, we prove the following

Theorem 3.1. Let $u_{0} \in L_{2}(0, L), \phi \in L_{2}(0, T), \bar{u} \in Y$, and the hypotheses $(9,10)$ be satisfied with $\left(C_{1}-\right.$ $\left.C_{0}\right) /\left(C_{1}+C_{0}\right)+k_{2} L<1$. Then the problem (15) has a unique solution $\tilde{u} \in Y$.

Proof. To solve the problem (15), we consider the following iterative process:

$$
-\left(\tilde{u}^{(n+1)}, \frac{\partial w}{\partial t}\right)+\left(\tilde{u}^{(n+1)}, A w\right)+\left(F\left(\tilde{u}^{(n)}+\bar{u}\right), w\right)=q(w), \forall w \in W_{T},
$$

with an initial approximation $\tilde{u}^{(0)} \in Y$. The remainder $v^{(n)}=\tilde{u}^{(n+1)}-\tilde{u}^{(n)}$ is the solution to the linear problem of the form (22), then using Lemmas 3.3 and 3.2, we obtain the estimate for $\left\|v^{(n)}\right\|_{Y}$ :

$$
\left\|v^{(n)}\right\|_{Y} \leq\left\|F\left(\tilde{u}^{(n)}+\bar{u}\right)-F\left(\tilde{u}^{(n-1)}+\bar{u}\right)\right\|_{W_{T}^{*}} \leq k\left\|v^{(n-1)}\right\|_{Y},
$$

with the constant $k$ defined in (21).

By applying successively the inequality (25) we show that the following estimate holds for any integers $n$ and $m$ :

$$
\left\|\tilde{u}^{(n+m)}-\tilde{u}^{(n)}\right\|_{Y} \leq a \frac{k^{n}-k^{n+m}}{1-k},
$$

where $a=\left\|\tilde{u}^{(0)}\right\|_{Y}+\left\|F\left(\tilde{u}^{(0)}+\bar{u}\right)\right\|_{W_{T}^{*}}$.

By the hypothesis, $k<1$, therefore, the inequality (26) implies that the sequence $\tilde{u}^{(n)}$ is convergent in $Y$. Hence, there exists an element $\tilde{u} \in Y$ such that $\lim _{n \rightarrow \infty} \tilde{u}^{(n)}=\tilde{u}$. Going to the limit for $m \rightarrow \infty$ in (26), we get the following estimate for the convergence rate:

$$
\left\|\tilde{u}-\tilde{u}^{(n)}\right\|_{Y} \leq a \frac{k^{n}}{1-k}
$$

Since $\tilde{u}^{(n)} \rightarrow \tilde{u}$ as $n \rightarrow \infty$, and the operators of the problem (15) are bounded from $Y$ into $W_{T}^{*}$, it is easily seen that $\tilde{u}$ is a solution to the problem (15).

Let us show that the solution of the problem (15) is unique. Suppose there exist two solutions $\tilde{u}_{1}$ and $\tilde{u}_{2}$ to the problem (15). Then we obtain the following problem for the remainder $\tilde{u}_{1}-\tilde{u}_{2}$ :

$$
-\left(\tilde{u}_{1}-\tilde{u}_{2}, \frac{\partial w}{\partial t}\right)+\left(\tilde{u}_{1}-\tilde{u}_{2}, A w\right)=-\left(F\left(\tilde{u}_{1}+\bar{u}\right), w\right)+\left(F\left(\tilde{u}_{2}+\bar{u}\right), w\right), w \in W_{T} .
$$

Using Lemmas 3.3 and 3.2 , we get

$$
\left\|\tilde{u}_{1}-\tilde{u}_{2}\right\|_{Y} \leq k\left\|\tilde{u}_{1}-\tilde{u}_{2}\right\|_{Y} .
$$

If $k<1$, the inequality (28) may hold only when $\tilde{u}_{1}=\tilde{u}_{2}$. This ends the proof. 
Remark 3.4. The hypothesis $\bar{u} \in Y$ is satisfied if, for example, $u_{0} \in L_{2}(0, L), u_{1}=0, f \in W_{T}^{*}$.

The condition of the boundedness of the coefficients $C(u), \tilde{K}(u), \frac{\partial \tilde{K}}{\partial u}$ are satisfied for some applied problems, the problems of the soil water movement among them [16].

The iterative process (24) is well-known as the successive approximation method; it may be used for numerical solution of the problem. Its convergence rate is defined by the formula (27).

\section{The PRoblem of DATA ASSimilation}

In this section, we consider the following data assimilation problem: find the initial condition $\theta_{0}$ and the solution $\theta$ such that

$$
\frac{\partial \theta}{\partial t}-\frac{\partial}{\partial z} D \frac{\partial \theta}{\partial z}+\frac{\partial K}{\partial z}=0, t \in(0, T),\left.z \in(0, L) \theta\right|_{t=0}=\theta_{0},\left.\theta\right|_{z=L}=\theta_{1},\left.\left(D \frac{\partial \theta}{\partial z}-K\right)\right|_{z=0}=\phi\left(\left.\theta\right|_{z=0}\right)
$$

$$
J\left(\theta_{0}\right)=\inf _{v} J(v)
$$

where

$$
J\left(\theta_{0}\right)=\frac{\varepsilon}{2}\left\|\theta_{0}\right\|_{X}^{2}+\frac{1}{2} \int_{0}^{T}\left\|\theta-\theta^{o}\right\|_{X}^{2} \mathrm{~d} t
$$

$\varepsilon \geq 0$ is the regularization parameter, $\theta^{\circ}$ is the observational data function, $\phi$ is the nonlinear function arisen from (5), $X=L_{2}(0, L)$ is the space introduced in Section 3.

On the basis of Theorem 3.1, we can prove the solvability of the problem $(29,30)$. Below, we assume all the hypotheses of Section 3 be satisfied, and $\phi \equiv \phi(t) \in L_{2}(0, T)$. The following theorem holds.

Theorem 4.1. Let $\theta^{\circ} \in Y,\left(C_{1}-C_{0}\right) /\left(C_{1}+C_{0}\right)+k_{2} L<1$. Then, for $\varepsilon>0$, there exists a solution $\theta_{0} \in X$ to the problem (29) and (30).

Proof. Let $v_{n}$ be a sequence minimizing $J(v)$, i.e. $J\left(v_{n}\right) \rightarrow \inf _{v \in X} J(v), n \rightarrow \infty$. Since $J(v) \geq \frac{\varepsilon}{2}\|v\|_{X}^{2}, \forall v \in X$, then for $\varepsilon>0$, the sequence $v_{n}$ is bounded: $\left\|v_{n}\right\|_{X} \leq$ const. Hence, there exists a weakly convergent subsequence $v_{n}$ (we denote it also $v_{n}$ ). The Hilbert space $X$ is weakly closed, therefore, there exists an element $\bar{v} \in X$ such that $v_{n} \rightarrow \bar{v}$ (weakly in $X$ ), that is, $\left(v_{n}, p\right)_{X} \rightarrow(\bar{v}, p)_{X}, \forall p \in X$.

Let $\theta_{n}$ and $\theta$ be the solutions of the original problem (29) for $\left.\theta_{n}\right|_{t=0}=v_{n}$ and $\left.\theta\right|_{t=0}=\bar{v}$, respectively. Using the Kirchoff transformation $u_{n}=\int_{0}^{\theta_{n}} D(s) \mathrm{d} s, u=\int_{0}^{\theta} D(s) \mathrm{d} s$ and following the arguments of Section 3, we come [as in (15)] to the problem for the remainder:

$$
-\left(u_{n}-u, \frac{\partial w}{\partial t}\right)+\left(u_{n}-u, A w\right)+\left(F\left(u_{n}\right)-F(u), w\right)=q_{n}(w), \forall w \in W_{T},
$$

where $q_{n}(w)=\left.\alpha \int_{0}^{L}\left(v_{n}-\bar{v}\right) w\right|_{t=0} \mathrm{~d} z$. The problem (32) may be written in the form:

$$
\left(u_{n}-u,-\frac{\partial w}{\partial t}+A w+\left(F^{\prime}(\xi)\right)^{*} w\right)=q_{n}(w), \forall w \in W_{T},
$$

where $\xi \in Y$, and $\left(F^{\prime}(\xi)\right)^{*}: W_{T} \rightarrow Y$ is the operator adjoint to $F^{\prime}(\xi)$.

Consider $p \in Y$ and introduce the following auxiliary problem:

$$
\begin{gathered}
-\frac{\partial w}{\partial t}+A w+\left(F^{\prime}(\xi)\right)^{*} w=p \\
\left.w\right|_{t=T}=0 .
\end{gathered}
$$


Following the proof of Theorem 3.1, we can show that for any $p \in Y$ there exists a unique solution $w \in W_{T}$ to the problem (34), and

Hence,

$$
\|w\|_{W_{T}}=\left\|p-\left(F^{\prime}(\xi)\right)^{*} w\right\|_{Y} \leq\|p\|_{Y}+k\|w\|_{W_{T}} .
$$

$$
\|w\|_{W_{T}} \leq \frac{1}{1-k}\|p\|_{Y}, p \in Y
$$

Since $q_{n}(w) \rightarrow 0, n \rightarrow \infty$, then from (33), we get $\left(u_{n}-u, p\right) \rightarrow 0, p \in Y$. By this is meant that $u_{n} \rightarrow u$ (weakly in $Y$ ), and, therefore, $\theta_{n} \rightarrow \theta$ (weakly in $Y$ ). The functional $S(\cdot)=\|\cdot\|^{2}$ is known [18] to be lower semi-continuous in the weak topology, then

$$
\lim \inf J\left(v_{n}\right) \geq J(\bar{v})
$$

and, therefore,

$$
\inf _{v \in X} J(v) \geq J(\bar{v})
$$

Hence,

$$
\inf _{v \in X} J(v)=J(\bar{v})
$$

that is, $\bar{v}$ gets the minimum to the functional $J(v)$. This proves the theorem.

The necessary optimality condition [18] reduces the problem $(29,30)$ to the following system for finding $\theta, \theta^{*}$, and $\theta_{0}$ :

$$
\begin{gathered}
\frac{\partial \theta}{\partial t}-\frac{\partial}{\partial z} D \frac{\partial \theta}{\partial z}+\frac{\partial K}{\partial z}=0, t \in(0, T),\left.z \in(0, L) \theta\right|_{t=0}=\theta_{0},\left.\theta\right|_{z=L}=\theta_{1},\left.\left(D \frac{\partial \theta}{\partial z}-K\right)\right|_{z=0}=\phi\left(\left.\theta\right|_{z=0}\right) \\
-\frac{\partial \theta^{*}}{\partial t}-D \frac{\partial^{2} \theta^{*}}{\partial z^{2}}-\frac{\partial K}{\partial \theta} \frac{\partial \theta^{*}}{\partial z}=\theta^{o}-\theta, t \in(0, T),\left.z \in(0, L) \theta^{*}\right|_{t=T}=0,\left.\theta^{*}\right|_{z=L}=0,\left.\left(D \frac{\partial \theta^{*}}{\partial z}-\phi^{\prime} \theta^{*}\right)\right|_{z=0}=0 \\
\nabla J\left(\theta_{0}\right) \equiv \varepsilon \theta_{0}-\left.\theta^{*}\right|_{t=0}=0 .
\end{gathered}
$$

The solvability of the original problem (35) has been investigated in Section 3. The adjoint problem (36) is a linear 1-D parabolic problem with the bounded coefficients and its solution properties are well-known [10]. The solvability of the whole system (35)-(37) follows from Theorem 4.1.

To solve (35)-(37) one may use the gradient methods, the gradient of functional $J$ being calculated successively by the formulas (35)-(37).

To be sure that the initial value function obtained with assimilation gets the unique minimum to the functional $J$ it is reasonable to use the second order adjoint analysis [29], considering the Hessian of the problem.

Let us rewrite the problem (35)-(37) in the operator form:

$$
\begin{gathered}
\frac{\partial \theta}{\partial t}=F(\theta), \quad t \in(0, T) \\
\left.\theta\right|_{t=0}=\theta_{0} \\
-\frac{\partial \theta^{*}}{\partial t}-\left(F^{\prime}(\theta)\right)^{*} \theta^{*}=\theta^{o}-\theta, \quad t \in(0, T) \\
\left.\theta^{*}\right|_{t=T}=0 \\
\varepsilon \theta_{0}-\left.\theta^{*}\right|_{t=0}=0,
\end{gathered}
$$

where $F(\theta),\left(F^{\prime}(\theta)\right)^{*}$ are the corresponding operators of the problems $(35,36)$.

After solving the system (38)-(40), we get three functions $\theta, \theta^{*}$ and $\theta_{0}$. Then, the Hessian $H\left(\theta_{0}\right)$ of the problem is defined successively by the following steps [29]: 
1) for a given $v \in X$ find $\psi$ as the solution to the problem

$$
\begin{gathered}
\frac{\mathrm{d} \psi}{\mathrm{d} t}=F^{\prime}(\theta) \psi \\
\left.\psi\right|_{t=0}=v
\end{gathered}
$$

2) using $\psi$ and $\theta^{*}$, find $\psi^{*}$ as the solution of the adjoint problem:

$$
-\frac{\mathrm{d} \psi^{*}}{\mathrm{~d} t}-\left(F^{\prime}(\theta)\right)^{*} \psi^{*}=\left[\frac{\partial^{2} F}{\partial \theta^{2}} \psi\right]^{*} \theta^{*}-\psi,\left.\psi^{*}\right|_{t=T}=0 ;
$$

3) put

$$
H\left(\theta_{0}\right) v=\varepsilon v-\left.\psi^{*}\right|_{t=0} .
$$

The Hessian $H\left(\theta_{0}\right)$ is symmetric. To study its positiveness, consider the scalar product $\left(H\left(\theta_{0}\right) v, v\right)$ in $X$. By definition, we have

$$
\left(H\left(\theta_{0}\right) v, v\right)=\left(\varepsilon v-\left.\psi^{*}\right|_{t=0}, v\right)=\varepsilon(v, v)-\left(\left.\psi^{*}\right|_{t=0},\left.\psi\right|_{t=0}\right) .
$$

Since the problem (43) is adjoint to $(42)$, we get

$$
\left(\left.\psi^{*}\right|_{t=0},\left.\psi\right|_{t=0}\right)=\int_{0}^{T}\left(\psi,\left(\frac{\partial^{2} F}{\partial \theta^{2}} \psi\right)^{*} \theta^{*}-\psi\right) \mathrm{d} t .
$$

Hence,

$$
\left(H\left(\theta_{0}\right) v, v\right)=\varepsilon(v, v)+\int_{0}^{T}(\psi, \psi) \mathrm{d} t-\int_{0}^{T}\left(\frac{\partial^{2} F}{\partial \theta^{2}} \psi \psi, \theta^{*}\right) \mathrm{d} t .
$$

The function $\psi$ is the solution to the linear problem (41)-(42). It is known [26] that the following estimate for $\psi$ is valid:

$$
\int_{0}^{T}\|\psi\|^{2} \mathrm{~d} t \leq M(v, v)
$$

where

$$
M=\int_{0}^{T} \mathrm{e}^{-\int_{0}^{t} \lambda_{\min }(\tau) \mathrm{d} \tau},
$$

and $\lambda_{\min }$ is the lower bound of the spectrum of the operator $F^{\prime}(\theta)+\left(F^{\prime}(\theta)\right)^{*}$.

If the operator $\left(\frac{\partial^{2} F}{\partial \theta^{2}} \circ\right)^{*} \theta^{*}$ is bounded, i.e.

$$
\left\|\left(\frac{\partial^{2} F}{\partial \theta^{2}} \psi\right)^{*} \theta^{*}\right\| \leq h\|\psi\|, \quad h=\text { const }>0
$$

then,

$$
\left|\int_{0}^{T}\left(\frac{\partial^{2} F}{\partial \theta^{2}} \psi \psi, \theta^{*}\right) \mathrm{d} t\right| \leq h \int_{0}^{T}\|\psi\|^{2} \mathrm{~d} t \leq h M(v, v),
$$

and

$$
\left(H\left(\theta_{0}\right) v, v\right) \geq(\varepsilon-h M)(v, v),
$$

i.e. for $h M<\varepsilon$ the operator $H\left(\theta_{0}\right)$ is positive definite. 
The inequality (47) is satisfied for our model under the hypothesis that the solutions $\theta, \theta^{*}$ of the problems (35, 36) are regular enough. Thus, for instance, when the coefficients $D$ and $K$ are taken in the form (3a), we get

$$
\left\|\left(\frac{\partial^{2} F}{\partial \theta^{2}} \psi\right)^{*} \theta^{*}\right\|_{Y}=\beta\left\|D \frac{\partial^{2} \theta^{*}}{\partial z^{2}} \psi+K^{\prime} \frac{\partial \theta^{*}}{\partial z} \psi\right\|_{Y}=\beta\left\|\left(\theta^{o}-\theta\right) \psi+\frac{\partial \theta^{*}}{\partial t} \psi\right\|_{Y} \leq h\|\psi\|_{Y},
$$

where $h=\beta\left(\left\|\theta-\theta_{o}\right\|_{L_{\infty}}+\left\|\frac{\partial \theta^{*}}{\partial t}\right\|_{L_{\infty}}\right)$, and $L_{\infty}=L_{\infty}((0, T) \times(0, L))$.

The condition $h M<\varepsilon$ may be verified in calculations. However, one should remember that this condition is only sufficient, and in practice it may turn more preferable to compute the eigenvalues of the Hessian by the technique of [29].

\section{NumericAl RESUltS}

The soil water assimilation is studied numerically in this section. The following experiments belong to identical twin framework: observation is generated from the model and the optimal initial field is retrieved by seeking the minimum of the cost function (31) with $\varepsilon=0$.

The numerical model is designed by finite difference scheme, and its resolution is $\Delta z=0.05 \mathrm{~m}$; the time step is $\Delta t=0.6 \mathrm{~min}$. In variational data assimilation, the cost function is taken as

$$
J_{d}\left(\theta_{0}\right)=\frac{1}{2} \sum_{k=0}^{K}\left\langle\theta_{k}-\theta_{k}^{o}, \theta_{k}-\theta_{k}^{o}\right\rangle,
$$

where $\theta_{k}$ is model state at $t=k \Delta t$ and $\theta_{k}^{o}$ the corresponding observed data at the same time.

\subsection{The evaluation of VDA system}

The assimilation is realized through searching the minimum of the cost function iteratively by a descent gradient method. The gradient can be got from the backward integration of the adjoint model and its accuracy should be checked before used in the optimization algorithm. Here the gradient check criteria is taken as Navon et al. [23]:

$$
R(\alpha)=\frac{J(x+\alpha \nabla J /\|\nabla J\|)-J(x)}{\alpha\|\nabla J\|},
$$

The check result is as following:

\begin{tabular}{|c|c|}
\hline$\alpha$ & $\mathrm{R}$ \\
\hline $1.000000000000000 \mathrm{E}-001$ & 1.467237924961184 \\
\hline $1.000000000000000 \mathrm{E}-002$ & 1.064499862508060 \\
\hline $1.000000000000000 \mathrm{E}-003$ & 1.006638330725731 \\
\hline $1.000000000000000 \mathrm{E}-004$ & 1.000663935343485 \\
\hline $1.000000000000000 \mathrm{E}-005$ & 1.000066412649290 \\
\hline $1.000000000000000 \mathrm{E}-006$ & 1.000006640769757 \\
\hline $1.000000000000000 \mathrm{E}-007$ & 1.000000665333111 \\
\hline $1.000000000000000 \mathrm{E}-008$ & 1.000000147527289 \\
\hline $1.000000000000000 \mathrm{E}-009$ & $9.999998988580549 \mathrm{E}-001$ \\
\hline $1.000000000000000 \mathrm{E}-010$ & $9.999871876912144 \mathrm{E}-001$ \\
\hline
\end{tabular}

This verifies that the gradient obtained from the adjoint model is correct and the minimization procedure M1QN3 [7] is applied in our study. 


\subsubsection{Assimilation with perfect data}

Here, we want to know if the initial field is very noisy, can it be adjusted from the good observation? The initial reference state, superposed on a Gaussian distributed random error is used as the first guess. The magnitude of the error is about $20 \%$ of the one of the initial reference state. The assimilation window is 24 hours, and the data are provided every 6 hours.

Case 1. The lower part of soil is wetter than the upper. The numerical result is shown in Figure 1, where Figure 1a gives the initial reference state, and the $x$-axis is soil wetness; Figure 1b presents the change of cost function, and the $x$-axis is iterative step number; Figure $1 c$ gives the variation of the norm of the gradient of the functional $J_{d}$ with the iterative step number.

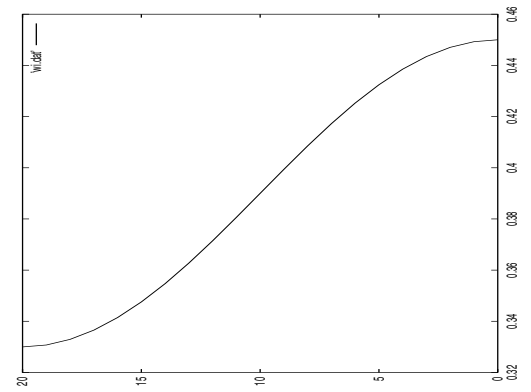

(a)

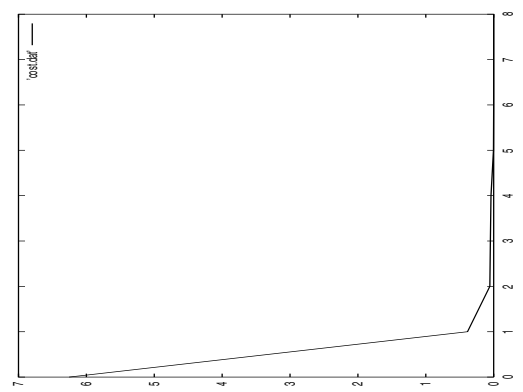

(b)

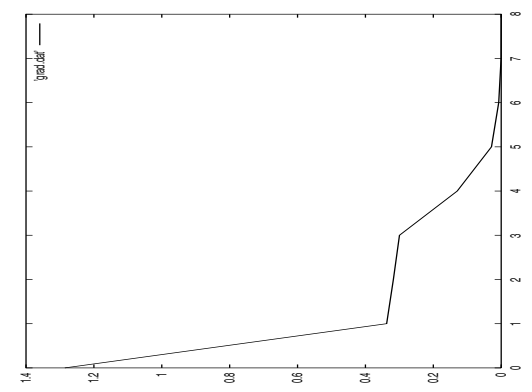

(c)

Figure 1. In case 1, (a) the initial reference state; (b) the variation of cost function; (c) the variation of gradient norm.

Case 2. The upper part of soil is wetter than the lower. The numerical result is shown in Figure 2, whose interpretation being the same as for Figure 1.

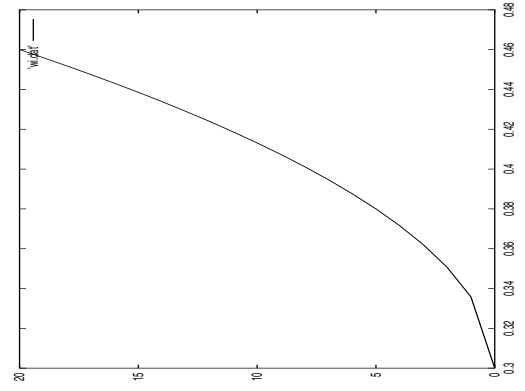

(a)

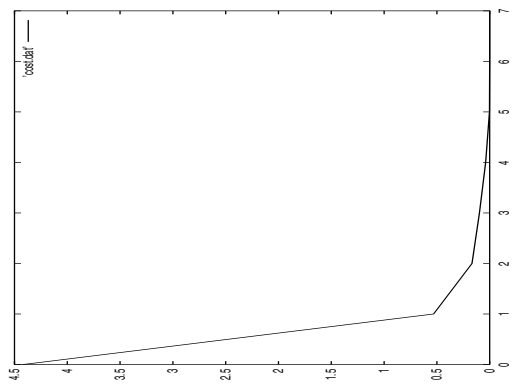

(b)

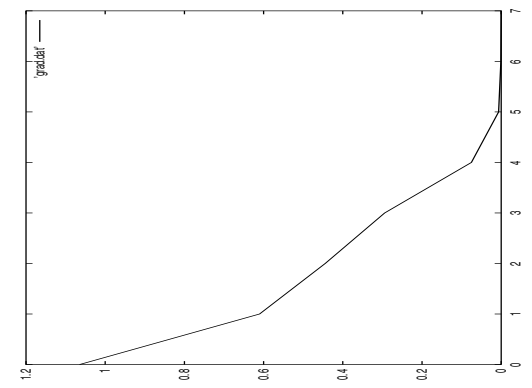

(c)

FIGURE 2. In case 2, (a) the initial reference state; (b) the variation of cost function; (c) the variation of gradient norm.

\subsubsection{Assimilation with noisy data}

The previous experiments demonstrate that the model's assimilation ability is acceptable. In fact, the observations are always not perfect. So, more experiments should be carried out for situations with noisy data. The following is in this line. The model generated the reference state. Then, the reference state with a $5 \%$ 
Gaussian random perturbation is taken as the observation. That is, the observation error is not zero as the above.

Case 3. The reference state at $6 \mathrm{~h}$ is taken as the first guess field for assimilation. The result is shown in Figure 3, where Figure 3a gives the initial reference state of soil wetness; Figure 3b and Figure 3c present the change of cost function and gradient norm with the iterative step number, respectively.

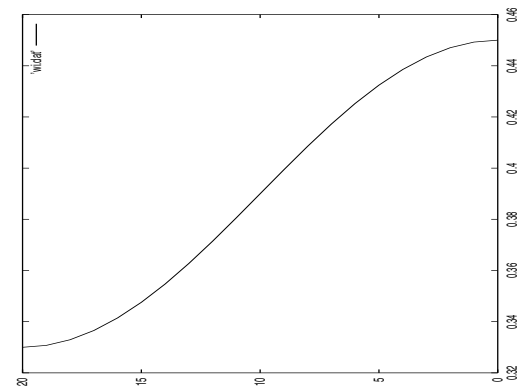

(a)

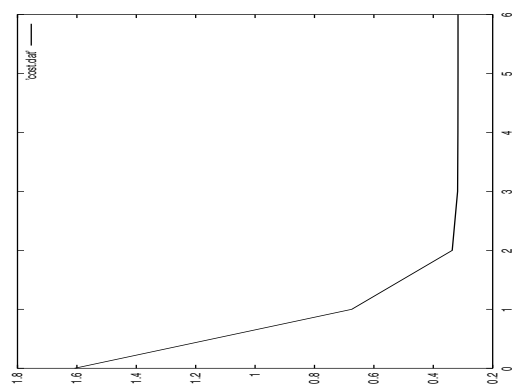

(b)

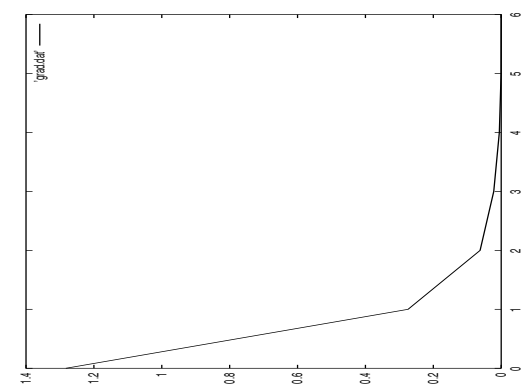

(c)

FiguRE 3. In case 3, (a) the initial reference state; (b) the variation of cost function; (c) the variation of gradient norm.

Case 4. The reference state at $12 \mathrm{~h}$ is taken as the initial guess for assimilation. The minimization progress is presented in Figure 4, whose interpretation being the same as for Figure 3.
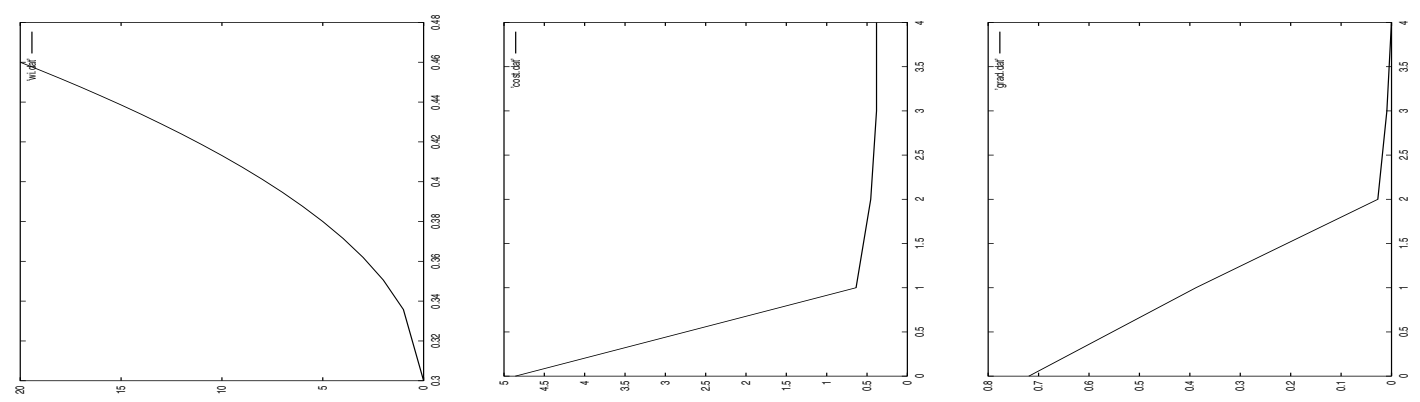

Figure 4. In case 4, (a) the initial reference state; (b) the variation of cost function; (c) the variation of gradient norm.

From these experiments, we can see that the assimilation technique works well for the soil water movement model under consideration. Further, the uniqueness of the assimilation result and the convergence rate of the minimization progress is studied in the context of the second order adjoint.

\subsection{The uniqueness and convergence rate analysis}

Gill et al. [8] pointed out that the convergence rate of minimization process is related to the conditional number of the Hessian matrix of the cost function: the larger the conditional number is, the lower the convergence rate. This problem has been widely investigated in the atmospheric field $[5,6,17,29]$. It is also clear that if the Hessian is positive, the solution of the optimal control problem is unique [14,29]. In this soil water assimilation problem, as noted in Section 4, the Hessian $H\left(\theta_{0}\right)$ is a real symmetric matrix in practice. So all of its eigenvalues can be calculated out by Jacobi method and the uniqueness of the solution and the convergence rate of cost function can be studied together. 
The construction of the second order adjoint model can be found in [13]. The assimilation window is 24 hours, and the observation interval $\left(\Delta T^{\mathrm{obs}}\right)$ is $12,6,3,2$ and $1 \mathrm{~h}$, respectively. The formula of the cost function is the same as (51). An even soil moisture state is taken as the first guess for each case. In the experiments, the minimal eigenvalue and the conditional number of the Hessian matrix are calculated and the total number of iterative steps noted. The result is in the following table:

\begin{tabular}{|c|c|c|c|}
\hline $\begin{array}{c}\text { Observation } \\
\text { interval }\end{array}$ & $\begin{array}{c}\text { Minimal } \\
\text { eigenvalue }\end{array}$ & $\begin{array}{c}\text { Conditional } \\
\text { number }\end{array}$ & $\begin{array}{c}\text { Number of } \\
\text { iteration }\end{array}$ \\
\hline $12 \mathrm{~h}$ & 0.212 & 432.5 & 7 \\
\hline $6 \mathrm{~h}$ & 0.219 & 451.1 & 11 \\
\hline $3 \mathrm{~h}$ & 0.226 & 497.3 & 17 \\
\hline $2 \mathrm{~h}$ & 0.231 & 547.6 & 18 \\
\hline $1 \mathrm{~h}$ & 0.210 & 815.2 & 22 \\
\hline
\end{tabular}

It can be seen that in each case, the minimal eigenvalue of the Hessian is greater than zero. This means all of the eigenvalues are positive, as well as the Hessian. So it can be concluded the assimilation solution is unique. Comparing the conditional number and the number of iteration, the relationship that the latter increasing larger according to the former is clear. Further, considering the observation interval, it can be found that the more frequent the observed data used, the more the time cost. This demonstrates the intuitive opinion that using the observation as much as possible in assimilation is not appropriate in view of practice.

The variation of cost function and norm of gradient during the minimization progress in each case is shown in the following figure.

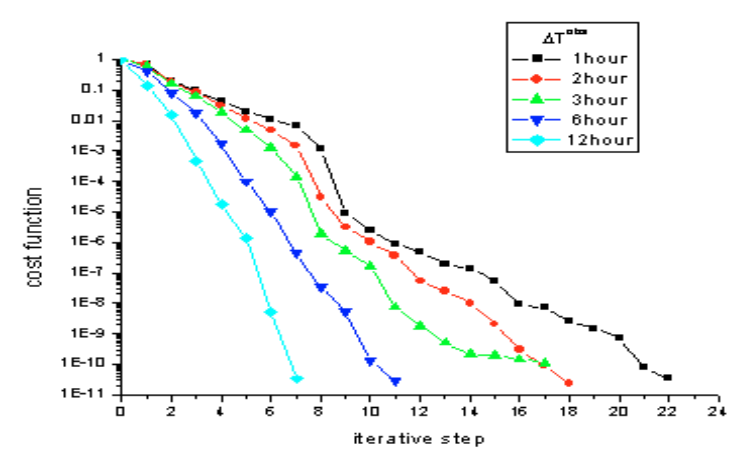

(a)

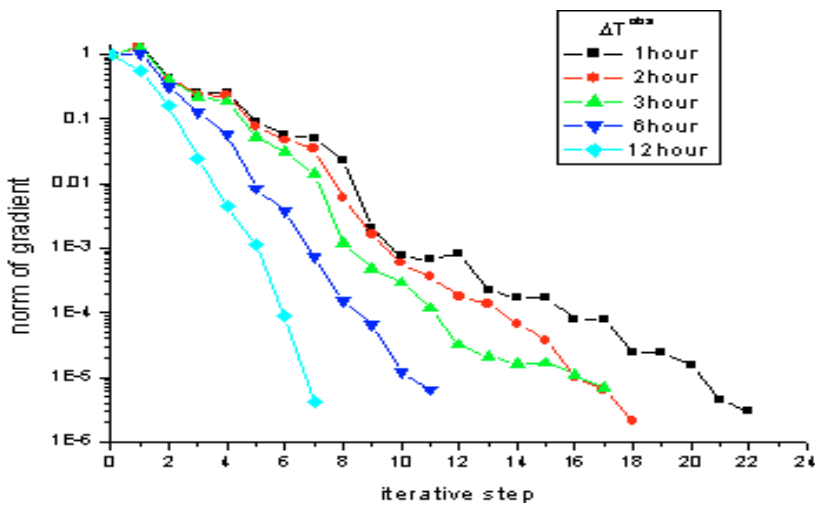

(b)

FIgURE 5. The variation of cost function (a) and norm of gradient (b) with the iterative step number. 


\section{REFERENCES}

[1] V.I. Agoshkov and A.P. Mishneva, Calculation of the diffusion coefficient in a nonlinear parabolic equation. Preprint of the Department of Numerical Mathematics, USSR Acad. Sci., Moscow (1988), No. 200.

[2] V.I. Agoshkov and G.I. Marchuk, On the solvability and numerical solution of data assimilation problems. Russ. J. Numer. Anal. Math. Modelling 8 (1986) 1-16.

[3] H.W. Alt and S. Luckhaus, Quasilinear elliptic-parabolic differential equations. Math. Zeitschrift 183 (1983) $311-341$.

[4] E. Blayo, J. Blum and J. Verron, Assimilation variationnelle de données en océanographie et réduction de la dimension de l'espace de contrôle. Équations aux Dérivées Partielles et Applications (Articles dédiées à Jacques-Louis Lions) (1998) 205-219.

[5] W.C. Chao and L.P. Chang, Development of a four-dimensional variational analysis system using the adjoint method at GLA. Part I: Dynamics. Mon. Wea. Rev. 120 (1992) 1661-1673.

[6] J.C. Derber, Variational four-dimensional analysis using quasigeostrophic constraints. Mon. Wea. Rev. 115 (1987) $998-1008$.

[7] J.-C. Gilbert and C. Lemarechal, Some numerical experiments with variable storage quasi-Newton algorithms. Math. Program. B25 (1989) 408-435.

[8] P.E. Gill, W. Murray and M.H. Wright, Practical Optimization. Academic Press (1981).

[9] D. Henry, Geometric Theory of Semilinear Parabolic Equations. New York, Springer (1981).

[10] O.A. Ladyzhenskaya and N.N. Uraltseva, A survey on solvability of boundary value problems for uniformly elliptic and parabolic equations of the second order. Uspekhi Math. Nauk 41 (1986) 59-83.

[11] O.A. Ladyzhenskaya, V.A. Solonnikov and N.N. Uraltseva, Linear and Quasilinear Parabolic Equations. Moscow, Nauka (1967).

[12] M.M. Lavrentiev, A priori Estimates and Existence Theorems for Nonlinear Parabolic Equations. Novosibirsk, Nauka (1982).

[13] F.-X. Le Dimet and I. Charpentier, Méthodes de second ordre en assimilation de données. Équations aux Dérivées Partielles et Applications (Articles dédiées à Jacques-Louis Lions) (1998) 623-639.

[14] F.-X. Le Dimet, H.E. Ngodock and B. Luong, Sensitivity analysis in variational data assimilation. J. Met. Soc. Japan 75 (1997) 245-255.

[15] F.-X. Le Dimet and O. Talagrand, Variational algorithms for analysis and assimilation of meteorological observations: theoretical aspects. Tellus A $\mathbf{3 8}$ (1986) 97-110.

[16] Zh. Lei and Sh. Yang, The Dynamics of Soil Water. Tsinghua University Press (1986).

[17] Y. Li, I.M. Navon, W. Yang, X. Zou, J.R. Bates, S. Moorthi and R.W. Higgins, Four-dimensional variational data assimilation experiments with a multilevel semi-Lagrangian semi-implicit general circulation model. Mon. Wea. Rev. 122 (1994) $966-983$.

[18] J.-L. Lions, Optimal Control of Systems Governed by Partial Differential Equations. New York, Springer (1970).

[19] J.-L. Lions, Some Methods for Solving Nonlinear Problems. Moscow, Mir (1972).

[20] J.-L. Lions and E. Magenes, Problémes aux limites non homogènes et applications. Paris, Dunod (1968).

[21] G.I. Marchuk, V.I. Agoshkov and V.P. Shutyaev, Adjoint Equations and Perturbation Algorithms in Nonlinear Problems. CRC Press Inc. New York (1996).

[22] M. Mu, Global smooth solutions of two-dimensional Euler equations. Chin. Sci. Bull. 35 (1990) 1895-1900.

[23] I.M. Navon, X. Zou, J. Derber and J. Sela, Variational data assimilation with an adiabatic version of the NMC spectral model. Mon. Wea. Rev. 120 (1992) 1433-1446.

[24] O.A. Oleinik and E.V. Radkevich, Method of introducing a parameter for study of evolution equations. Uspehi Math. Nauk 33 (1978) $7-76$.

[25] V. Penenko and N.N. Obraztsov, A variational initialization method for the fields of meteorological elements. Meteorol. Gidrol. 11 (1976) 1-11.

[26] V.P. Shutyaev, Some properties of the control operator in the problem of data assimilation and iterative algorithms. Russ. J. Numer. Anal. Math. Modelling 10 (1995) 357-371.

[27] T.I. Zelenyak, M.M. Lavrentiev and M.P. Vishnevski, Qualitative Theory of Parabolic Equations. Utrecht, VSP Publishers (1997).

[28] T.I. Zelenyak and V.P. Michailov, Asymptotical behaviour of solutions of mathematical physics. Partial Diff. Eqs. (1970) 96-110.

[29] X. Zou, I. Navon and F.-X. Le Dimet, Incomplete observations and control of gravity waves in variational data assimilation. Tellus A 44 (1992) 273-296. 